\title{
Stability is realization-dependent: some examples
}

\author{
Hans Zwart ${ }^{\mathrm{a}, *}$, Yutaka Yamamoto ${ }^{\mathrm{b}}$, Yasuhisa Gotoh ${ }^{\mathrm{c}}$ \\ ${ }^{a}$ Department of Applied Mathematics, University of Twente, P.O. Box 217, 7500 AE Enschede, The Netherlands \\ ${ }^{\mathrm{b}}$ Division of Applied Systems Science, Faculty of Engineering, Kyoto University, Kyoto 606, Japan \\ 'Department of Mathematics, Faculty of Science, Kyoto University, Kyoto 606, Japan
}

(Received 31 August 1993; revised 5 December 1993)

\begin{abstract}
This paper gives some examples with the same impulse response, both approximately controllable and observable, but one of them is exponentially stable and the other is unstable. Some related spectral properties are also investigated.
\end{abstract}

Keywords: Realization; Stability; Shift semigroup; Spectrum

\section{Introduction}

It is well understood that for finite-dimensional systems, under the key assumption of controllability and observability, there is no gap between external and internal notions of stability. For example, if a transfer matrix belongs to $H_{\infty}$, then its controllable and observable realization is stable and vice versa. Based on this fact, for the study of robust stability of this class of systems, one often considers unstructured uncertainty which involves a certain norm bound for frequency response, and then stability is automatically assured by examining transfer functions.

Such a general norm bound condition usually allows nonrational perturbations, so even when we deal with a finite-dimensional plant, the robust stability question involves an infinite-dimensional question. However, it is well known $[1,4]$ that for a given transfer function, there are in general many nonisomorphic approximately controllable and observable realizations. On the other hand, it is definitely desirable to be able to conclude exponential stability rather than a mere $L_{2}$-input/output stability; unknown initial states can be guaranteed to decay to zero if an internal stability property is present. While stability is primarily a topological notion, we do not particularly specify the state space topology when we discuss robust stability from the input/output viewpoint. Hence the clearly desirable situation is that the stability notion is independent among all approximately controllable and observable realizations, thereby bypassing the problem of aforementioned nonuniqueness of realizations. On the other hand, if we want some notion of internal stability to guarantee the effects by the unknown initial states to decay in time, and if the desirable property above does not hold, it would mean that we have to be more careful in dealing with robust stability question with unstructured uncertainty.

\footnotetext{
* Corresponding author.
} 
The objective of this paper is to show that the latter is indeed the case: we give some elementary examples that the notion of stability crucially depends on the choice of state space topology. In fact, we give some impulse responses where one approximately controllable and observable realization is exponentially stable and the other is unstable.

Notation and convention. As usual, $L_{2}(a, b)$ is the space of Lebesgue square integrable functions on $(a, b)$. Likewise, $L_{2}^{\text {loc }}[0, \infty)$ is the space of functions on $[0, \infty)$ that are square integrable on every compact interval. In what follows, $\sigma_{t}$ denotes the left shift operator: $\left(\sigma_{t} \varphi\right)(\tau):=\varphi(\tau+t)$. This gives a $C_{0}$-semigroup in $L_{2}(0, \infty)$ whose infinitesimal generator is $(\mathrm{d} / \mathrm{d} t)$ with as domain all absolutely continuous function whose derivative is in $L_{2}(0, \infty)$.

For a given function $\varphi$, its $L_{2}(a, b)$ norm will be denoted by $\|\varphi\|_{L_{2}(a, b)}$. Likewise, $\|x\|_{Z}$ denotes the norm of $x$ in a Hilbert space $Z$. For a given continuous linear map $T: Z \rightarrow W$, its operator norm will be denoted by $\|T\|_{W / Z}$, when precise distinction is desirable. For the subset $E$ in a linear space $Z$, span $E$ denotes the smallest subspace that contains $E$.

$\mathbb{C}_{\beta}^{+}$denotes the right-half plane: $\{s \in \mathbb{C} \mid \operatorname{Re} s>\beta\}$, whereas $\mathbb{C}_{\beta}^{-}$denotes the left-half plane: $\{s \in \mathbb{C} \mid \operatorname{Re} s<\beta\}$.

\section{Counterexamples via shift realizations}

We construct our counterexamples in the following way: We take the left shift semigroup $\left\{\sigma_{t}\right\}$ as the transition semigroup over two spaces; one is $Z=L_{2}(0, \infty)$ and the other, say $W$, is $Z$ with a different topology with completion. The idea is to show that (i) it is possible to enforce different growth rates on the same shift semigroup $\left\{\sigma_{t}\right\}$ by changing topologies, and (ii) both systems can be made approximately controllable and observable.

To be more precise, define the following subspace of $L_{2}^{\text {loc }}[0, \infty)$ :

$$
W:=\left(\left.\varphi \in L_{2}^{\text {loc }}[0, \infty)\left|\int_{0}^{\infty}\right| \varphi(\tau)\right|^{2} \mathrm{e}^{-2 \tau} \mathrm{d} \tau<\infty\right\} .
$$

Clearly this is the completion of $L_{2}(0, \infty)$ with respect to the inner product

$$
\langle\varphi, \psi\rangle_{\boldsymbol{W}}:=\int_{0}^{\infty} \varphi(\tau) \overline{\psi(\tau)} \mathrm{e}^{-2 \tau} \mathrm{d} \tau
$$

and $\left\{\sigma_{t}\right\}$ comprise a $C_{0}$-semigroup in $W$. On its growth rate, we have the following lemma.

Lemma 2.1. The semigroup $\sigma_{t}$ in $W$ grows as fast as $\mathrm{e}^{t}$, i.e., $\left\|\sigma_{t}\right\|_{W / W}=\mathrm{e}^{t}$.

Proof. For any $f \in W$, we have

$$
\int_{0}^{\infty}\left|\left(\sigma_{t} \varphi\right)(\tau)\right|^{2} \mathrm{e}^{-2 \tau} \mathrm{d} \tau=\int_{0}^{\infty}|\varphi(\eta)|^{2} \mathrm{e}^{-2(\eta-t)} \mathrm{d} \eta=\int_{0}^{\infty}|\varphi(\eta)|^{2} \mathrm{e}^{2 t} \mathrm{e}^{-2 \eta} \mathrm{d} \eta \leqslant \mathrm{e}^{2 t}\|\varphi\|_{W}^{2},
$$

so that $\left\|\sigma_{t}\right\|_{W / W} \leqslant \mathrm{e}^{t}$. On the other hand, if $\operatorname{supp} \varphi \subset(t, \infty)$, then the equality holds in the inequality above. Therefore, we must have $\left\|\sigma_{t}\right\|_{w / W}=\mathrm{e}^{t}$.

Let us now state the following lemma.

Lemma 2.2. There exists a function $b \in L_{2}(0, \infty)$ such that $\overline{\operatorname{span}_{\tau} \geqslant 0\left\{\sigma_{\tau} b\right\}}=L_{2}(0, \infty)$.

Proof. A direct construction of such a $b$ can be found in [10]. The existence follows also from the results in $[3,5]$, since the Laplace transform of a $b$ satisfying the conditions in the lemma is a cyclic vector. 
Example 2.3. Consider the weighted shift semigroup $\mathrm{e}^{-(1 / 2) t} \sigma_{t}$ in two spaces $Z=L_{2}(0, \infty)$ and $W$ as defined in (1). Consider the two systems given in $Z$ and $W$ described by (essentially) the same functional differential equation

$$
\frac{\mathrm{d} z_{t}}{\mathrm{~d} t}=A z_{t}+b u(t)
$$

and the observation equation

$$
y(t)=c z_{t}:=z_{t}(0),
$$

where $A$ is the infinitesimal generator (in $Z$ or in $W$ ) of the semigroup $\mathrm{e}^{-(1 / 2) t} \sigma_{t}, c: C[0, \infty) \mapsto \mathbb{R}$, and $b \in Z$ is the element given in Lemma 2.2 .

Let $\Sigma_{Z}(A, b, c)$ be the system with state space $Z$, and $\Sigma_{W}(A, b, c)$ that with $W$. Since the observation operator in both systems is the point observation, they are clearly both observable. Furthermore, the set of approximately reachable elements is precisely the closure of $\operatorname{span}\left\{\sigma_{t} b ; t \geqslant 0\right\}$ (in each space). It is known by Lemma 2.2 to be dense in $Z$. Since $Z=L_{2}(0, \infty)$ is clearly dense in $W$, this set is also dense in $W$, completing the proof for approximate controllability of both systems.

Now we claim the following:

$$
\left\|\mathrm{e}^{-(1 / 2) t} \sigma_{t}\right\|_{Z / Z}=\mathrm{e}^{-(1 / 2) t}, \quad\left\|\mathrm{e}^{-(1 / 2) t} \sigma_{\imath}\right\|_{W / W}=\mathrm{e}^{(1 / 2) t},
$$

that is, $\Sigma_{Z}(A, b, c)$ is exponentially stable and $\Sigma_{W}(A, b, c)$ is unstable. However, this is readily obvious from $\left\|\sigma_{t}\right\|_{z / Z}=1$ and $\left\|\sigma_{t}\right\|_{W / W}=\mathrm{e}^{t}$.

In other words, we have started out with the same state transition equation, considered it in two different spaces, make it approximately controllable and observable in both spaces, but the resulting two systems present totally different stability behavior. In other words, the notion of stability is dependent on the state space topology.

Via direct calculation, we see that the transfer function of both systems is $\hat{b}\left(s+\frac{1}{2}\right)$, the Laplace transform of $b$. On the other hand, the spectrum of the first system is $\overline{\mathbb{C}_{-1 / 2}^{-}}$while that for the second system is $\overline{\mathbb{C}_{1 / 2}^{-}}$. The first is readily obvious; the second statement follows from the fact that we can allow a function that grows as fast as $\mathrm{e}^{(1-\varepsilon) t}$ for any $\varepsilon>0$ as an element in $W$. Therefore, the singularities of the transfer function can be quite irrelevant to the spectrum of a realization.

Remark 2.4. An abstract example of two approximately controllable and observable discrete-time systems yielding the same transfer function, but with different spectrum is also given by Fuhrmann [4, p. 176]. Since in his example the generators are bounded, they satisfy the spectrum determined growth condition, i.e., stability is fully determined by the location of spectrum. Therefore, although it is not stated explicitly there, we see that this also gives a discrete-time example of two systems where stability notion is not preserved.

\section{Counterexample with discrete spectrum}

In the previous section we have seen that it is possible that a transfer function has a stable and an unstable realization. This shows that approximate controllability and observability have little control over the stability property. Also, in the previous examples, even the spectrum of the system is not preserved, and they do not even coincide with the poles of the transfer function. In many realizations the spectrum coincides with the poles of the transfer function. Hence, a natural question is if the stability property of a realization is the same among all approximately controllable and observable realizations which have the same spectrum. Example 3.6 will show that the answer to this question is again negative. 
Definition 3.1. For $\alpha>0$ we define the following linear spaces:

$$
Z_{\alpha}:=\left\{\left(z_{n}\right)_{n \in \mathbb{N}} \mid z_{n}=\left(z_{n 1}, \ldots, z_{n n}\right)^{\mathrm{T}} \in \mathbb{C}^{n} \text { and } \sum_{n=1}^{\infty} \sum_{k=1}^{n}\left|z_{n k} \alpha^{-n+k}\right|^{2}<\infty\right\} .
$$

On this space we define the following inner product.

$$
\langle z, w\rangle_{\alpha}:=\sum_{n=1}^{\infty} \sum_{k=1}^{n} z_{n k} \overline{w_{n k}} \alpha^{2(-n+k)} .
$$

Hence we regard $z$ as an infinitely long vector of the following form:

$$
z=\left(z_{11}, z_{21}, z_{22}, z_{31}, \ldots, z_{33}, \ldots, \ldots, z_{n 1}, \ldots, z_{n n}, \ldots, \ldots, \ldots\right)^{\mathrm{T}} .
$$

It is easy to show that $Z_{\alpha}$ with the inner product defines a Hilbert space. On this Hilbert space we define the following operator.

Definition 3.2. Let $\lambda_{n}$ be a sequence of pure imaginary numbers such that $\lambda_{n} \neq \lambda_{m}$ for $n \neq m$ and they have no finite accumulation point. For $z=\left(z_{n}\right) \in Z_{\alpha}$ and $t \in \mathbb{R}$ we define

$$
T(t) z=\left(T_{n}(t) z_{n}\right),
$$

where

$$
T_{n}(t)=\mathrm{e}^{\lambda_{n} t}\left(\begin{array}{cccc}
1 & t & \cdots & \frac{t^{n-1}}{(n-1) !} \\
0 & 1 & & \vdots \\
\vdots & & \ddots & t \\
0 & \cdots & 0 & 1
\end{array}\right) .
$$

We have to assure that the above given expression for $T(t)$ is well defined on $Z_{\alpha}$. In the next lemma we show that $T(t)$ defines a $C_{0}$-semigroup on $Z_{\alpha}$.

Lemma 3.3. The operator defined by (8) is a $C_{0}$-semigroup on $Z_{\alpha}$ with group bound $1 / \alpha$.

Proof. From [8] it is well known that $T(t)$ defines a $C_{0}$-semigroup on $Z_{1}$ with growth bound 1 . We shall use this result to prove the assertion.

Let $z$ be an element of $Z_{\alpha}$ and denote $y=T(t) z$. Then by the definition of $T(t)$ and (9) we have that

$$
y_{n k}=\mathrm{e}^{\lambda_{n} t} \sum_{l=k}^{n} \frac{t^{l-k}}{(l-k) !} z_{n l}, \quad n \geqslant 1,1 \leqslant k \leqslant n .
$$

Calculating the norm of this element gives

$$
\begin{aligned}
\|y\|_{\alpha}^{2} & =\langle y, y\rangle_{\alpha}=\sum_{n=1}^{\infty} \sum_{k=1}^{n}\left|y_{n k} \alpha^{-n+k}\right|^{2} \quad\left(\text { since } \lambda_{n} \in J \mathbb{R}\right) \\
& =\sum_{n=1}^{\infty} \sum_{k=1}^{n}\left|\sum_{l=k}^{n} \frac{t^{l-k}}{(l-k) !} z_{n l} \alpha^{-n+k}\right|^{2}=\sum_{n=1}^{\infty} \sum_{k=1}^{n}\left|\sum_{l=k}^{n} \frac{t^{l-k} \alpha^{k-l} \alpha^{-n+l}}{(l-k) !} z_{n l}\right|^{2} \\
& =\sum_{n=1}^{\infty} \sum_{k=1}^{n}\left|\sum_{l=k}^{n} \frac{(t / \alpha)^{l-k}}{(l-k) !} z_{n l} \alpha^{-n+l}\right|^{2}=\left\|T\left(\frac{t}{\alpha}\right) \tilde{z}\right\|_{1}^{2}
\end{aligned}
$$


where $\tilde{z}=\left(\tilde{z}_{11}, \tilde{z}_{21}, \ldots, \ldots\right)^{\mathrm{T}}$ with $\tilde{z}_{n l}=z_{n l} \alpha^{-n+l}$. Using the results from [8] we conclude that

$$
\|y\|_{\alpha}=\left\|T\left(\frac{t}{\alpha}\right) \tilde{z}\right\|_{1} \leqslant \mathrm{e}^{t / \alpha}\|\tilde{z}\|_{1}=\mathrm{e}^{t / \alpha}\left[\sum_{n=1}^{\infty} \sum_{l=1}^{n}\left|z_{n l} \alpha^{-n+l}\right|^{2}\right]^{1 / 2}=\mathrm{e}^{t / \alpha}\|z\|_{\alpha} .
$$

Hence $T(t)$ defines a bounded operator on $Z_{\alpha}$.

Since $T_{n}(t)$ is equal to the exponential of the $n \times n$ matrices consisting of one Jordan block, it is clear that $T(t)$ defines a $C_{0}$-semigroup on the dense set $\left\{z=\left(z_{n}\right) \mid z_{n}=0\right.$ for almost all $\left.n\right\}$. Using the fact that $T(t)$ is a bounded operator it is now easy to see that $T(t)$ defines a $C_{0}$-semigroup on $Z_{\alpha}$.

From (10) we conclude that the growth bound of $T(t)$ on $Z_{\alpha}$ is less or equal to $1 / \alpha$. The equality follows as in [8].

As in [8] we have that the infinitesimal generator is given by

$$
A z=\left(A_{n} z_{n}\right),
$$

where $A_{n}$ is the Jordan block of size $n \times n$ with $\lambda_{n}$ on the diagonal. Furthermore, the spectrum of this operator is exactly $\left\{\lambda_{n}\right\}$ and for $s \neq \lambda_{n}$ we have that

$$
(s I-A)^{-1} z=\left(\left(s I-A_{n}\right)^{-1} z_{n}\right),
$$

where

$$
\left(s I-A_{n}\right)^{-1}=\left(\begin{array}{cccc}
\frac{1}{s-\lambda_{n}} & \frac{1}{\left(s-\lambda_{n}\right)^{2}} & \cdots & \frac{1}{\left(s-\lambda_{n}\right)^{n}} \\
0 & \frac{1}{s-\lambda_{n}} & & \vdots \\
\vdots & & \ddots & \frac{1}{\left(s-\lambda_{n}\right)^{2}} \\
0 & \cdots & 0 & \frac{1}{s-\lambda_{n}}
\end{array} \mid .\right.
$$

Since the proof is the same as in [8], it is omitted here. From the fact that the $\left|\lambda_{n}\right| \rightarrow \infty$ as $n \rightarrow \infty$ and from the special form of $A$ it follows easily that the resolvent of $A$ is compact.

We have given a $C_{0}$-semigroup and state spaces such that the growth depends on the choice of the state space. It remains to give input and output operators such that the same transfer function is realized. In order to prove approximate controllability and observability, we need the following result of Zwart [9, Theorem IV.6].

Lemma 3.4. Let $V$ be a closed subspace of $Z_{\alpha}$. The following assertions are equivalent.

(1) $V$ is $T(t)$-invariant, i.e. $T(t) V \subset V$ for all $t \geqslant 0$;

(2) there exists $V_{n} \subset \mathbb{C}^{n}$ with $A_{n} V_{n} \subset V_{n}$ and $V=\sum_{n=1}^{\infty} V_{n}$.

Here we have identified a subspace $V_{n}$ of $\mathbb{C}^{n}$ with a subspace of $Z$ via the cononical injection.

With this lemma we can characterize all input and output operators, such that the associated systems are approximately controllable and observable. Let $A$ be the infinitesimal generator of $T(t)$ on $Z_{\alpha}$, and let $b, c \in Z_{\alpha}$. With these operators we can define the following system $\Sigma(A, b, c)$ given by

$$
\dot{z}(t)=A z(t)+b u(t), \quad y(t)=\langle z(t), c\rangle_{\alpha} .
$$

Lemma 3.5. Consider the system $\Sigma(A, b, c)$ on the state space $Z_{\alpha}$ as defined in (14), where $A$ denotes the infinitesimal generator of $T(t)$ as given in (11) and $b, c \in Z_{\alpha}$.

(1) The system $\Sigma(A, b,-)$ is approximately controllable if and only if $b_{n n} \neq 0$ for all $n \geqslant 1$.

(2) The system $\Sigma(A,-, c)$ is approximately observable if and only if $c_{n 1} \neq 0$ for all $n \geqslant 1$. 
Proof. The proof is based on the fact that the reachable subspace and the unobservable subspace are $T(t)$-invariant. We shall only give the proof of the second assertion, since the proof of the first one is similar.

A system is approximately observable if and only if the unobservable subspace is the zero subspace. The unobservable subspace $\mathscr{N O}$ is the largest $T(t)$-invariant subspace contained in the kernel of the output operator. Thus by Lemma 3.4 we have that $\mathscr{N O}=\sum_{n=1}^{\infty} \mathscr{N} \mathcal{O}_{n}$ with $\mathscr{N} \mathcal{O}_{n} \subset \mathbb{C}^{n}$, and $A_{n} \mathscr{N O} \mathcal{O}_{n} \subset \mathcal{N} \mathcal{O}_{n}$.

Since $\mathscr{N O}$ is contained in the kernel of $C=\langle\cdot, c\rangle_{\alpha}$, we have that $\mathcal{N} \mathcal{O}_{n}$ is contained in the kernel of $C_{n}=\left\langle\cdot, \tilde{c}_{n}\right\rangle_{\mathfrak{C}^{n}}$, where $\tilde{c}_{n k}=c_{n k} \alpha^{2(-n+k)}$. Hence, if $\mathscr{N} \mathcal{O}_{n}$ is nonzero, then the system $\Sigma\left(A_{n},-, \tilde{c}_{n}\right)$ is not observable. It is easy to see that if $\Sigma\left(A_{n},-, \tilde{c}_{n}\right)$ is not observable for some $n$, then the big system $\Sigma(A,-, c)$ is not observable. An initial state which is unobservable for the subsystem is unobservable for the whole system either. Hence we have that $\Sigma(A,-, C)$ is not observable if and only if there exists an $n$ such that the finite-dimensional system $\Sigma\left(A_{n},-, \tilde{c}_{n}\right)$ is not observable.

From finite-dimensional system theory it is well known that $\Sigma\left(A_{n},-, \tilde{c}_{n}\right)$ is observable if and only if $\tilde{c}_{n 1} \neq 0$. Hence, the system $\Sigma(A,-, c)$ is observable if and only if $c_{n 1} \neq 0$.

Now we can give a transfer function which admits a stable and an unstable approximately controllable and observable realization.

Example 3.6. Consider the transfer function

$$
f(s)=\sum_{n=1}^{\infty} \frac{a_{n}}{\left(s-\gamma_{n}\right)^{n}}
$$

where $\gamma_{n}=-\frac{3}{4}+\lambda_{n}$, and $\lambda_{n}$ are purely imaginary numbers with $\lambda_{n} \neq \lambda_{m}$ for $n \neq m$ and no finite accumulation points. Furthermore, we assume that $a_{n} \neq 0$ and $\left\{\sqrt{a_{n}} 2^{2(n-1)}\right\}$ is a square summable sequence. Under these assumptions it is easy to see that $f$ is a meromorphic function on $\mathbb{C}$.

We shall prove that the following systems are both approximately controllable and observable realizations of $f(s)$.

- On the state space $Z_{1}$ we define the system $S_{1}(t)=\mathrm{e}^{-(3 / 4) t} T(t), b^{1}=\left(b_{n}^{1}\right)$ with $b_{n}^{1}=\left(0, \ldots, 0, \sqrt{a_{n}}\right)^{\mathrm{T}}$ and $c^{1}=\left(c_{n}^{1}\right)$ with $c_{n}^{1}=\left(\sqrt{a_{n}}, 0, \ldots, 0\right)^{\mathrm{T}}$.

- On the state space $Z_{2}$ we define the system $S_{2}(t)=\mathrm{e}^{-(3 / 4) t} T(t), b^{2}=\left(b_{n}^{2}\right)$ with $b_{n}^{2}=\left(0, \ldots, 0, \sqrt{a_{n}}\right)^{\mathrm{T}}$ and $c^{2}=\left(c_{n}^{2}\right)$ with $c_{n}^{2}=\left(\sqrt{a_{n}} 2^{2(n-1)}, 0, \ldots, 0\right)^{\mathrm{T}}$.

From Lemma 3.3 we know that $S_{1}(t)$ is unstable and $S_{2}(t)$ is exponentially stable. Since $S_{1}(t)$ and $S_{2}(t)$ are just $T(t)$ multiplied with $\mathrm{e}^{-(3 / 4) t}$, it is easy to see that both systems are approximately controllable and observable if and only if $\Sigma\left(A, b^{1}, c^{1}\right)$ and $\Sigma\left(A, b^{2}, c^{2}\right)$ are approximately controllable and observable. By our assumption on the sequence $\left\{a_{n}\right\}$ and Lemma 3.5 this follows directly.

Let $A_{1}$ and $A_{2}$ denote the infinitesimal generators of $S_{1}(t)$ and $S_{2}(t)$, respectively. Then we have that for $\alpha=1$ or 2

$$
\begin{aligned}
\left\langle\left(s I-A_{\alpha}\right)^{-1} b^{\alpha}, c^{\alpha}\right\rangle_{\alpha} & =\left\langle((s-3 / 4) I-A)^{-1} b^{\alpha}, c^{\alpha}\right\rangle_{\alpha} \\
& \left.=\sum_{n=1}^{\infty} \frac{b_{n n}^{\alpha} c_{n 1}^{\alpha} \alpha^{2(-n+1)}}{\left(s-3 / 4-\lambda_{n}\right)^{n}} \quad \text { by }(7) \text { and }(13)\right) \\
& \left.=\sum_{n=1}^{\infty} \frac{a_{n}}{\left(s-3 / 4-\lambda_{n}\right)^{n}} \quad \text { (by the definition of } b_{n n}^{\alpha} \text { and } c_{n 1}^{\alpha}\right) \\
& =f(s) \quad(\text { by }(15)) .
\end{aligned}
$$

This shows that we have two approximately controllable and observable realizations of the same transfer functions, where one is exponentially stable and the other is unstable. Note that the generators in both cases have the same spectrum which equals the poles of the transfer function. 


\section{Concluding remarks}

We have shown examples in which the notion of stability crucially depends on the state space topology. It should then be interesting to ask the question: is there a reasonably large class of systems in which the stability notion is invariant among all approximately controllable and observable realizations? From Example 3.6 we see that this class is smaller than all meromorphic transfer functions. However, a recent manuscript [5] asserts that all Blaschke products are in this class. Unfortunately, many transfer functions are not Blaschke products. Transfer functions for which we like to know if they belong to the class are the pseudorational ones studied in, e.g., $[6,7]$. This is a topic for future study.

\section{Acknowledgment}

The second author is supported in part by the Tateishi Science and Technology Foundation. We want to thank Raimund Ober for sending us $[3,5]$.

\section{References}

[1] J.S. Baras, R.W. Brockett and P.A. Fuhrmann, State-space models for infinite-dimensional systems, IEEE Trans. Automat. Control AC-19 (1974) 693-700.

[2] P.J. Davis, Interpolation and Approximation (Dover, New York, 1975.)

[3] R.G. Douglas, H.S. Shapiro and A.L. Shields, Cyclic vectors and invariant subspaces for the backward shift operator, Ann. Inst. Fourier (Grenoble) 20 (1970) 37-76.

[4] P.A. Fuhrmann, Linear Systems and Operators in Hilbert Space (McGraw-Hill, New York, 1981.)

[5] R. Ober and Y. Wu, Infinite dimensional continuous time linear systems: stability and structure analysis, Technical Report \# 1, Center for Engineering Mathematics, The University of Texas at Dallas (1993).

[6] Y. Yamamoto, Pseudo-rational input/output maps and their realizations: a fractional representation approach to infinitedimensional systems, SIAM J. Control Optim. 26 (1988) 1415-1430.

[7] Y. Yamamoto, Equivalence of internal and external stability for a class of distributed systems, MCSS 4 (1991) $391-409$.

[8] J. Zabczyk, A Note on $C_{0}$-semigroups, Bull. l'Acad. Pol. de Sc. Serie Math. 23 (1975) 895-898.

[9] H.J. Zwart, Geometric Theory for Infinite-Dimensional Systems, Lecture Notes in Control and Information Sciences, Vol. 115 (Springer, Berlin, 1989).

[10] H. Zwart, Y. Yamamoto and Y. Gotoh, On the Stability Uniformity of Infinite-Dimensional Systems, in: Proc. 10th Internat. Conf. on Analysis and Optimization of Systems: State and Frequency Domain Approach for Infinite-Dimensional Systems, Lecture Notes in Control and Information Sciences, Vol. 185 (Springer, Berlin, 1992). 